\title{
Functionality of the Tumor Suppressor microRNA-1 in Malignant Tissue and Cell Line Cells of Uterine Leiomyosarcoma
}

\author{
MATTHIAS B. STOPE ${ }^{1}$, VICTOR CERNAT ${ }^{2}$, ANNE KAUL ${ }^{2}$, KAROLINE DIESING ${ }^{2}$, \\ DOMINIQUE KOENSGEN ${ }^{2}$, MARTIN BURCHARDT ${ }^{1}$ and ALEXANDER MUSTEA ${ }^{2}$
}

Departments of ${ }^{1}$ Urology and ${ }^{2}$ Gynecology and Obstetrics, University Medicine Greifswald, Greifswald, Germany

\begin{abstract}
Background/Aim: Uterine leiomyosarcoma $(u L M S)$ is a very rare mesenchymal tumor showing an aggressive clinical course and poor prognosis for patients. Due to the low incidence, little is known about molecular tumor biology and biomarkers of uLMS. Micro-RNA-1 (miR-1) has been identified as a pivotal tumor suppressor in numerous entities being suited as a molecular marker for tumor progression. Materials and Methods: uLMS patient samples were analyzed regarding their miR-1 expression levels. Furthermore, miR-1 growth inhibitory and target regulatory properties were examined in transfected uLMS cells SK-UT-1. Results: miR-1 was strongly suppressed in uLMS tumor tissue compared to adjacent healthy tissue. In vitro studies, however, failed to detect growth inhibitory properties of miR-1 in SK-UT-1 cells. The expression of the cell survival and MAP kinases Erk-1/2 and p38 was not targeted by miR-1. Conclusion: Tumor suppressive mechanisms of miR-1, seem to be inhibited in ULMS SK-UT-1 cells, maybe as part of the malignant transformation process. Regardless of the microRNA's cellular functionality, miR-1 may represent a promising biomarker of diagnosis in uLMS therapy.
\end{abstract}

Uterine leiomyosarcoma (uLMS) is a very rare and aggressive mesenchymal tumor which comprises about $1 \%$ of all uterine malignancies and one third of uterine sarcoma (1). In Germany, approximately 300 cases are diagnosed each year with a patient mean age of 50 years. Unfortunately, there exist no effective diagnostic techniques for identifying patients with uLMS. Even modern imaging scans using ultrasound, computed tomography (CT), and magnetic resonance imaging (MRI) fail to detect

Correspondence to: Matthias B. Stope, Department of Urology, University Medicine Greifswald, Ferdinand-Sauerbruch-Straße, 17475 Greifswald, Germany. Tel: +49 38348680436, Fax: +49 38348680435, e-mail: matthias.stope@uni-greifswald.de

Key Words: Uterine leiomyosarcoma, microRNA-1, tumor progression.
uLMS-specific signs (2). Consequently, uLMS is frequently discovered incidentally on histopathological analysis following hysterectomy.

Due to the aggressive clinical course of uLMS, patients have poor prognosis with early hematogenous spread and development of lung metastases within 2 years after primary therapy (2). Furthermore, the metastatic recurrence rate even in patients with localized early stage uLMS exceeds $50 \%$ according to the International Federation of Gynecology and Obstetrics (FIGO) (3).

Treatment of uLMS patients still remains challenging. Since response to chemo- and radiation therapy exhibits little, if any, effect, complete surgical resection represents the best and potentially curative treatment for localized uLMS $(2,4)$. Only a few prognostic factors (age, tumor size, early stage) have been evaluated for uLMS therapy, while molecular biomarkers are completely missing $(1,5)$.

MicroRNAs, such as microRNA-1 (miR-1), orchestrate crucial mechanisms in cancer progression and have moved into the focus of interest as novel biomarkers. $m i R-1$ has been identified as a tumor suppressor in numerous entities and is frequently down-regulated during cancer progression (6). In the study presented here, $m i R$-l's expression and functionality in uLMS was examined for the first time.

\section{Materials and Methods}

Patient samples. Tissue samples from uLMS patients $(n=4)$ and women without any history of cancer $(n=4)$ obtained from the Department of Gynecology and Obstetrics, University Medicine Greifswald, Greifswald, Germany, were analyzed. The presented study was approved by the Ethical Committee of the University Medicine Greifswald (registration no. III SV 05/04). All patients signed informed consent forms.

Cell culture. The uLMS cell line SK-UT-1 (Cell Lines Service, Eppelheim, Germany) was propagated in Dulbecco's modified Eagle medium F12 (Life Technologies, Darmstadt, Germany) supplemented with $10 \%$ fetal calf serum and $0.125 \%$ gentamicin in a humidified atmosphere at $37^{\circ} \mathrm{C}$ with $5 \% \mathrm{CO}_{2}$. 

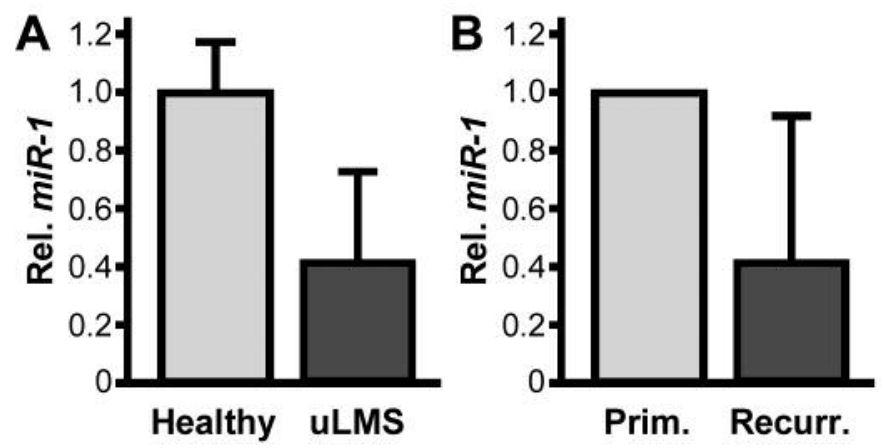

Figure 1. Expression analysis of miR-1 in uLMS tissue samples by quantitative RT-PCR. (A) miR-1 expression levels in uLMS patient samples (uLMS) compared to tissue samples from healthy donors (Healthy). (B) miR-1 expression levels in primary uLMS samples (Prim.) compared to samples from recurrent uLMS patients (Recurr.). Controls (Healthy, Prim.) were set to 1.0. Results are expressed as the mean $\pm S D$.

miR-1 overexpression experiments. SK-UT-1 cells were transiently transfected with the miR-1 encoding vector pmiR-1 (7). Transfection was performed using Lipofectamine2000 reagent (Life Technologies according to the manufacturer's protocol.

miR-1 quantification by reverse transcription-polymerase chain reaction. Total RNA was prepared using peqGOLDTrifast Reagent (Peqlab Biotechnology, Erlangen, Germany) according to the manufacturer's instructions. Subsequently, reverse transciption was perforemd with 100 ng RNA and Superscript II Reverse Transcriptase (Life Technologies), according to the protocol of Chen et al. (miR-1 stem-loop primer: 5'-GTCGTATCCAGTGCAGGGTCCGAGGTA TTCGCACTGGATACGACATACAT-3'; U6 stem-loop primer: 5'GTCATCCTTGCGCAGG-3') (8). For quantification, polymerase chain reaction (PCR) was performed on a CFX96 Real-Time System (Bio-Rad, München, Germany) with SensiMix SYBR hi-ROX Kit (Bioline, Luckenwalde, Germany) and specific primers: $m i R-1$ forward primer: 5'-GCCCGCTGGAATGTAAAGAA GTATG-3'; miR-1 reverse primer: 5'-GTGCAGGGTCCGAGGT-3'; U6 forward primer: 5'-CGCTTCGGCAGCACATATAC-3'; U6 reverse primer: 5'AGGGGCCATGCTAATCTTCT-3'. miR-1 signals were analyzed using the CFX Manager software (Bio-Rad) and standardized to U6 RNA as reference.

Proliferation assay. Cellular proliferation was examined by cell counting in a CASY Cell Counter (Roche Applied Science, Mannheim, Germany) with a capillary of $150 \mu \mathrm{m}$ in diameter. SKUT-1 cells were detached by trypsin treatment, resuspended in CASYton (1:100; Roche Applied Science, Mannheim, Germany) followed by three replicates of measurement.

Protein analysis by western blotting. Equal amounts of protein extracts in $50 \mathrm{mM}$ Tris- $\mathrm{HCl}, \mathrm{pH} 6.8,2.0 \% \mathrm{SDS}, 10 \%(\mathrm{w} / \mathrm{v})$ glycerol, $0.01 \%$ bromophenol blue, $5 \% \beta$-mercaptoethanol were separated by electrophoresis and blotted onto a polyvinylidene fluoride membrane (Millipore, Darmstadt, Germany). Subsequently, the membrane was incubated with antibodies directed against Erk-1/2 (Cell Signaling Technology, Danvers, MA, USA), p38 (Cell Signaling Technology), and $\beta$-Actin (Cell Signaling Technology) overnight and proteinspecific signals were detected by dye-conjugated secondary antibodies (LI-COR Biotechnology, Lincoln, NE, USA) in a LI-COR Odyssey Infrared Imager System (LI-COR Biotechnology, Lincoln,
NE, USA) according to the manufacturer's instructions. Protein levels were quantified using the Image Studio Lite Version 4.0 software (LICOR Biotechnology). $\beta$-Actin signals served as a loading control and protein expression levels were normalized to controls (control=1.0).

\section{Results}

The tissue expression level of miR-1 becomes down-regulated during uLMS progression. Analysis of uLMS patient's samples demonstrated a clear suppression of $m i R-1$ expression in uLMS cancer tissue. Levels of $m i R-1$ were decreased $2.4 \pm 1.2-$ fold in samples obtained from uLMS patients compared to healthy donors (Figure 1A). Further examination of the uLMS samples additionally revealed a $2.3 \pm 1.9$-fold diminished expression of miR-1 in uLMS tissue after recurrence compared to the primary uLMS material (Figure 1B). The limited quantity of patient samples, however, prohibited further statistical analysis of the obtained data.

Overexpression of miR-1 in an in vitro uLMS model failed to demonstrate anti-proliferative effects of the microRNA. To provide some insights on the role of $m i R-1$ in the molecular and cellular biology of uLMS cells, overexpression experiments were performed applying the established uLMS cell line SK-UT-1 (Figure 2A). Transiently transfected SKUT-1 cells demonstrated a 4.6 \pm 1.9 -fold overexpression of miR-1 levels compared to vector transfected control cells (Figure 2B). Despite the elevated levels of the tumor suppressor, in SK-UT-1 cells, proliferation was not affected in the presence of increased $m i R-1$ concentrations compared to mock-transfected control cells (Figure 2C).

The miR-1 targets Erk-1/2 and p38 are not affected in uLMS cells overexpressing $m i R-1$. Among others, $m i R-1$ 's tumor suppressive capacity is effective towards two pivotal targets: the extracellular signal-regulated kinases 1 and 2 (Erk-1/2) as well as the mitogen-activated protein (MAP) kinase p38. 


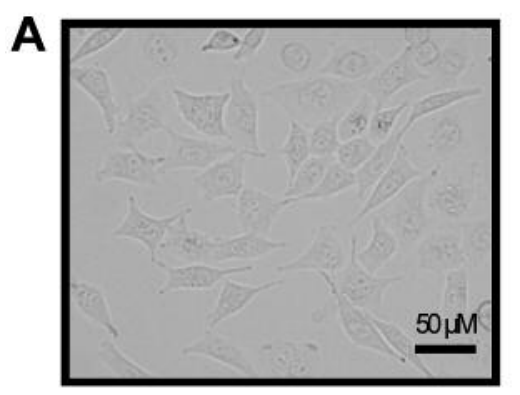

SK-UT-1 cells

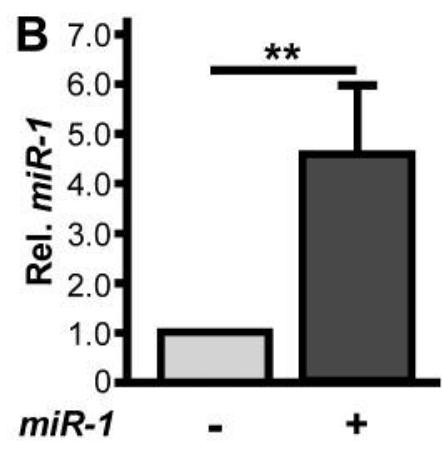

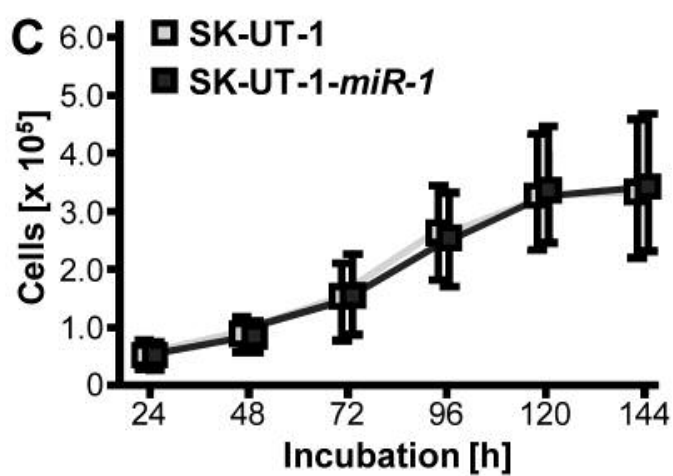

Figure 2. Transfection and cell growth analysis of the uLMS cell line SK-UT-1. (A) Microscopy of SK-UT-1 cells (magnification x20). (B) miR-1 overexpression (miR-1 +) compared to mock-transfected control cells (miR-1 -). (C) Cell growth analysis of SK-UT-1 cells overexpressing miR-1 (SK-UT-1-miR-1) compared to maternal control cells (SK-UT-1). Results are expressed as the mean $\pm S D$.
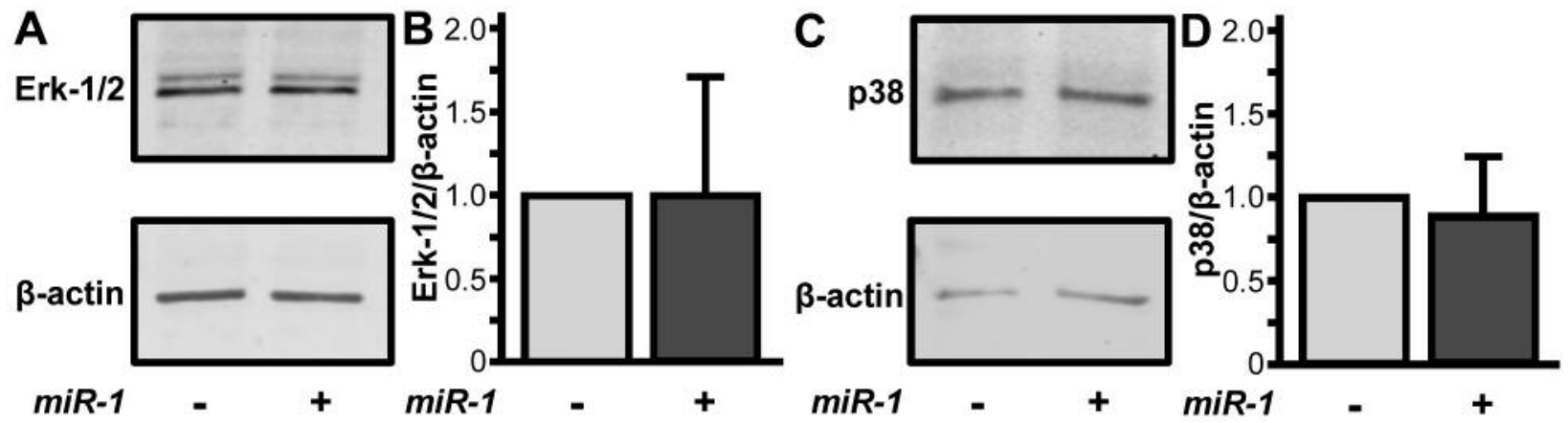

Figure 3. Protein expression analysis of the miR-1 targets Erk-1/2 and p38. Western blot analysis of Erk-1/2 (A) and p38 (C) and subsequent quantification of the Erk-1/2 (B) and p38 (D) expression. Controls (miR-1 -) were set to 1.0 and compared to miR-1 overexpression (miR-1 +). Results are expressed as the mean $\pm S D$.

A molecular study on $m i R-1$ dependent regulation of Erk-1/2 and p38 in transfected SK-UT-1 cells demonstrated no impact of the microRNA regarding the expression level of both survival kinases (Figure 3A and C). Quantification of the western blot analysis revealed, that neither Erk-1/2 (no reduction; Figure 3B) nor p38 (1.2 \pm 3 .3-fold reduction; Figure 3D) were efficiently targeted by overexpressed $m i R-1$.

\section{Discussion}

Studies evaluating the expression of $m i R-l$ in cancer confirmed significantly decreased amounts of $m i R-1$ compared to adjacent healthy tissue. This has been demonstrated in numerous entities including prostate cancer, bladder cancer, colon cancer, lung cancer, and osteosarcoma (reviewed in (6)). Although the sample size in the presented study prohibited statistical analysis, the data pointed to a strong suppression of miR-l in uLMS development and progression. uLMS is a rare gynecologic malignancy comprising less than $1 \%$ of all uterine cancers. Thus, clinical as well as experimental research on this disease is complicated and frequently fails, due to extremely low numbers of patient samples.

The examination of uLMS tissue revealed suppressed miR-1 levels, as expected. Following in vitro studies, however, failed to detect growth inhibitory properties of $m i R-1$ in SK-UT-1 cells. Generally, $m i R-l$ bears anti-proliferative and pro-apoptotic properties and is considered as a pivotal tumor suppressor in cancer cells $(7,9,10)$. Here, growth inhibitory effects are primarily determined by suppression of cell survival and MAP kinases $(9,11-13)$. However, after transfection of SK-UT-1 cells, neither Erk-1/2 nor p38 kinase expression was inhibited by elevated $m i R-1$ concentrations. On the one hand, this may represent a regulatory resistance against the tumor suppressor $m i R-1$ and occured accessorily to the reduction in miR- 1 expression. On the other hand, this could be certainly explained 
by a unique characteristic of the SK-UT-1 cell line that was utilized in the molecular study presented here. Finally, the very low number of patients samples as well as performing molecular analysis with only one uLMS cell line is the limitation of this molecular study, but further examinations are in progress to clarify this open question.

In conclusion, the tumor suppressor miR-1 is strongly suppressed in uLMS tumor tissue compared to adjacent healthy tissue, suggesting a critical role in uLMS progression control. Tumor suppressive mechanisms of $m i R-1$, however, seem to be inhibited in uLMS cells SK-UT-1, maybe as part of the malignant transformation process. Regardless of the microRNA's cellular functionality, $m i R-1$ may represent a promising biomarker for diagnosis of uLMS.

\section{References}

1 Cuppens T, Tuyaerts S and Amant F: Potential therapeutic targets in uterine sarcomas. Sarcoma 2015: 243298, 2015.

2 Denschlag D, Thiel FC, Ackermann S, Harter P, Juhasz-Boess I, Mallmann P, Strauss H-G, Ulrich U, Horn L-C, Schmidt D, Vordermark D, Vogl T, Reichardt P, Gass P, Gebhardt M and Beckmann MW: Sarcoma of the uterus. Guideline of the DGGG (S2k-Level, AWMF Registry No. 015/074, August 2015). Geburtshilfe Frauenheilkd 75: 1028-1042, 2015.

3 Zivanovic O, Jacks LM, Iasonos A, Leitao MM, JR, Soslow RA, Veras E, Chi DS, Abu-Rustum NR, Barakat RR, Brennan MF and Hensley ML: A nomogram to predict postresection 5-year overall survival for patients with uterine leiomyosarcoma. Cancer 118: 660-669, 2012.

4 Seagle B-LL, Sobecki-Rausch J, Strohl AE, Shilpi A, Grace A and Shahabi S: Prognosis and treatment of uterine leiomyosarcoma: A National Cancer Database study. Gynecol Oncol 145: 61-70, 2017.

5 Garg G, Shah JP, Kumar S, Bryant CS, Munkarah A and Morris RT: Ovarian and uterine carcinosarcomas: a comparative analysis of prognostic variables and survival outcomes. Int J Gynecol Cancer 20: 888-894, 2010.

6 Weiss M, Brandenburg L-O, Burchardt $M$ and Stope MB: MicroRNA-1 properties in cancer regulatory networks and tumor biology. Crit Rev Oncol Hematol 104: 71-77, 2016.
7 Stope MB, Stender C, Schubert T, Peters S, Weiss M, Ziegler P, Zimmermann U, Walther $\mathrm{R}$ and Burchardt M: Heat-shock protein HSPB1 attenuates microRNA miR-1 expression thereby restoring oncogenic pathways in prostate cancer cells. Anticancer Res 34: 3475-3480, 2014.

8 Chen C, Ridzon DA, Broomer AJ, Zhou Z, Lee DH, Nguyen JT, Barbisin M, Xu NL, Mahuvakar VR, Andersen MR, Lao KQ, Livak KJ and Guegler KJ: Real-time quantification of microRNAs by stem-loop RT-PCR. Nucleic Acids Res 33: e179, 2005.

9 Nasser MW, Datta J, Nuovo G, Kutay H, Motiwala T, Majumder S, Wang B, Suster S, Jacob ST and Ghoshal K: Down-regulation of micro-RNA-1 (miR-1) in lung cancer. Suppression of tumorigenic property of lung cancer cells and their sensitization to doxorubicin-induced apoptosis by miR-1. J Biol Chem 283: 33394-33405, 2008.

10 Kojima S, Chiyomaru T, Kawakami K, Yoshino H, Enokida H, Nohata N, Fuse M, Ichikawa T, Naya M, Nakagawa M and Seki $\mathrm{N}$ : Tumour suppressors $m i R-1$ and miR-133a target the oncogenic function of purine nucleoside phosphorylase (PNP) in prostate cancer. Br J Cancer 106: 405-413, 2012.

11 Wu H, Huang C, Taki FA, Zhang Y, Dobbins DL, Li L, Yan H and Pan X: Benzo-alpha-pyrene induced oxidative stress in Caenorhabditis elegans and the potential involvements of microRNA. Chemosphere 139: 496-503, 2015.

12 Zhang X, Zheng Y, Jagadeeswaran G, Ren R, Sunkar R and Jiang H: Identification of conserved and novel microRNAs in Manduca sexta and their possible roles in the expression regulation of immunity-related genes. Insect Biochem Mol Biol 47: 12-22, 2014.

13 Xu L, Zhang Y, Wang H, Zhang G, Ding Y and Zhao L: Tumor suppressor miR-1 restrains epithelial-mesenchymal transition and metastasis of colorectal carcinoma via the MAPK and PI3K/AKT pathway. J Transl Med 12: 244, 2014. 